\title{
Tecnologias educacionais voltadas para gestantes: revisão integrativa
}

\author{
Educational technologies for pregnant women: integrative review
}

\section{Tecnologías educativas para mujeres embarazadas: revisión integradora}

Amanda Vitória Santos de Brito ${ }^{1}$, Janilson de Souza Menezes ${ }^{1}$, Matheus Lopes dos Santos ${ }^{1}$, Marlucilena Pinheiro da Silva ${ }^{1}$, Nely Dayse Santos da Mata ${ }^{1}$, Elizabeth Teixeira ${ }^{2}$, Camila Rodrigues Barbosa Nemer ${ }^{1 *}$.

\section{RESUMO}

Objetivo: Identificou-se as Tecnologias Educacionais propostas para gestantes, publicadas nos últimos cinco anos. Métodos: Tratou-se de uma revisão integrativa, realizada nas bases de dados Medline, Lilacs e Bdenf, no período de maio a junho de 2021. Os critérios de inclusão foram: artigos completos, disponíveis on-line, em português e/ou inglês e que abordaram a temática escolhida. O período para corte foi 2016 a 2021. Os critérios de exclusão: publicações na modalidade cartas, resenhas e editoriais; teses, dissertações, monografia; duplicados; sem convergência com a questão de pesquisa; cujo método não estava bem descrito; com texto completo indisponível; e que não foram produzidos para a realidade brasileira. Resultados: A amostra final foi constituída por 20 artigos, nos quais se destacou a desigualdade regional tanto de produção científica como de foco dos estudos, o uso de aplicativos móveis, lacunas de assuntos que precisam ser abordados pelas tecnologias voltadas para as gestantes e estas tecnologias estão em fase de aplicação ao público-alvo. Considerações finais: Ficou evidente um aumento de publicações e o protagonismo da enfermagem quanto a construção, validação e aplicação de Tecnologias Educacionais, entretanto, estas ainda são escassas no que tange à atenção ao pré-natal.

Palavras-chave: Cuidado pré-natal, Gestantes, Tecnologia educacional, Educação em saúde.

\section{ABSTRACT}

Objective: The Educational Technologies proposed for pregnant women, published in the last five years, were identified. Methods: This is an integrative review, carried out in the MEDLINE, LILACS and BDENF databases, from May to June 2021. Inclusion criteria were: complete articles, available online, in Portuguese and/or English and that addressed the chosen theme. The period for cutting was 2016 to 2021. Exclusion criteria: publications in the form letters, reviews and editorials; theses, dissertations, monographs; duplicates; no convergence with the research question; whose method is not well described; with full text unavailable; and that have not been produced for the Brazilian reality. Results: The final sample consisted of 20 articles, in which regional inequality was highlighted both in scientific production and in the focus of studies, the use of mobile applications, gaps in issues that need to be addressed by technologies aimed at pregnant women and that these technologies are being applied to the target audience. Final considerations: There is an increase in publications and the role of nursing in the construction, validation and application of Educational Technologies. However, they still need to grow in prenatal care.

Keywords: Prenatal care, Pregnant women, Educational technology, Health education.

\section{RESUMEN}

Objetivo: Se identificaron las Tecnologías Educativas propuestas para mujeres embarazadas, publicadas en los últimos cinco años. Métodos: Se trata de una revisión integradora, realizada en las bases de datos Medline, Lilacs y Bdenf, de mayo a junio de 2021. Los criterios de inclusión fueron: artículos completos, disponibles online, en portugués y / o inglés y que abordaran la temática elegida. El período de corte fue de 2016 a 2021. Criterios de exclusión: publicaciones en las cartas modelo, reseñas y editoriales; tesis, disertaciones, monografías; duplicados; sin convergencia con la pregunta de investigación; cuyo método no fue bien descrito; con texto completo no disponible; y que no fueron producidos para la realidad brasileña. Resultados: La muestra final estuvo conformada por 20 artículos, en los cuales se destacó la desigualdad regional tanto en la producción científica como en el enfoque de los estudios, el uso de aplicaciones móviles, brechas en temas que necesitan ser abordados por TE dirigidos a mujeres embarazadas y que estas tecnologías se están aplicando al público objetivo. Consideraciones finales: Se incrementan las publicaciones y el rol de la enfermería en la construcción, validación y aplicación de Tecnologías Educativas. Sin embargo, todavía necesitan crecer en atención prenatal.

Palabras clave: Atención prenatal, Mujeres embarazadas, Tecnología educativa, Educación para la salud.

\footnotetext{
1 Universidade Federal do Amapá (Unifap), Macapá - AP. *E-mail: camila.barbosa@unifap.br

2 Universidade Federal do Pará, Belém - PA.
}

Financiamento: Fundação de Amparo à Pesquisa do Estado do Amapá - Fundação Tumucumaque. Programa Pesquisa para o SUS: Gestão Compartilhada em Saúde - PPSUS. Processo individual: 250.203.024/2021 e Programa de Auxílio ao Pesquisador- PAPESQ/UNIFAP-2021. 


\section{INTRODUÇÃO}

Lima VK, et al. (2019), ressaltaram que é de extrema importância que, no decorrer das semanas de gestação, o trabalho da equipe da atenção básica com relação ao processo educação-saúde no pré-natal seja efetivo e busque sanar as dúvidas das gestantes, pois as diversas alterações sofridas pelas mulheres nesse período levam à eclosão de sentimentos como: medo, insegurança e ansiedade, que são comumente relacionados à expectativa da gravidez, às dúvidas sobre o processo do parto, puerpério e os cuidados com o recém-nascido. Logo, é imprescindível que a gestante disponha de apoio à saúde, de forma integral, visando sempre à qualidade de vida materno-infantil.

Jardim MJ, et al. (2019), referiram que é dever de todos os profissionais de saúde envolvidos na assistência ao pré-natal serem facilitadores das práticas de educação em saúde sempre visando aos fatores biopsicossociais, como forma de esclarecimento de dúvidas e orientação sobre os temores relacionados ao período gestacional, parto, pós-parto e puerpério. Essas medidas possibilitam que as mulheres busquem sua autonomia e empoderamento sobre essa fase importante em suas vidas.

Entre as várias mudanças que vêm acontecendo no mundo, Sabino LM, et al. (2016), citaram o avanço tecnológico como atividade que tem facilitado as ações diárias das pessoas. As transformações também ocorrem no campo da enfermagem, em que são aproveitadas as tecnologias em diferentes contextos, tanto assistencial quanto educacional, com oportunidades de implementar ou desenvolver as tecnologias para maior crescimento da profissão e melhora da relação entre enfermeiro e cliente. Onde destacaram o uso de tecnologias educacionais por esses profissionais, para a prestação do cuidado.

Diante das possíveis necessidades que possam enfrentar, Jardim MJ, et al. (2019), disseram que as mulheres gestantes contam com o protagonismo do profissional enfermeiro, como facilitador desse processo de empoderamento, como criador de tecnologias educativas, que busquem sanar dúvidas e visem à melhora na adesão ao pré-natal. Isso faz com que a relação enfermeiro-gestante seja fortalecida e possibilita uma melhor compreensão da sua clientela por parte do profissional.

As Tecnologias Educacionais (TEs), segundo Cassiano AN, et al. (2020), são ferramentas que possibilitam a ampliação do conhecimento, das habilidades, das atitudes e podem proporcionar autonomia, empoderamento e autoconhecimento dos sujeitos, comunidade, estudantes e profissionais de saúde, capazes também de influenciar a assunção da responsabilidade relacionada às práticas de ensino e do cuidado. Essas TEs têm características de apoio como: "aquisitiva de competências, mediadora de conhecimentos, promotora da saúde, útil para o cuidado e interativa". A utilização dessas TEs pode resultar em adoção de comportamentos saudáveis, reflexão das atitudes e práticas, promoção da adesão, uniformização e simplificação de acesso às informações e melhoria na qualidade de vida, entre outros benefícios.

Na enfermagem, as TEs podem se apresentar por meio de três tendências: tecnologias para a educação técnica e superior, com estudantes; tecnologias para a educação em saúde, com a comunidade; e tecnologias para a educação continuada, com profissionais. Salbego C, et al. (2018), ressaltaram que o ambiente hospitalar é lembrado pela utilização de ferramentas sofisticadas, as quais auxiliam nos procedimentos realizados por enfermeiros e outros profissionais de saúde. Nesse cenário, é possível perceber o uso de tecnologias sob uma visão de produto e/ou processo. Na tecnologia como produto, estão acercadas as informatizações, informações e artefatos; na tecnologia como processo, os artifícios pertinentes ao ensino e à aprendizagem do indivíduo, bem como os saberes e conhecimentos estruturados do homem, que podem aderir à criação de produtos.

As tecnologias educacionais em saúde (TEs) são ferramentas para desenvolver o trabalho educativo e o processo de cuidar, tanto no ambiente hospitalar quanto na atenção básica. No Brasil, o Sistema Único de Saúde (SUS) disponibiliza materiais educativos impressos (cartilhas, folhetos, manuais etc.) para uso na assistência ao pré-natal, porém, geralmente, esses materiais são de conteúdo denso e com abordagem geral, sendo um entrave na utilização e compreensão, principalmente para pessoas que possuem baixo nível de escolaridade (INDARA CB, et al., 2018).

Mostrou-se a necessidade de propor estudos que abordem a temática, fazendo um levantamento do que já se tem produzido acerca das TEs voltadas a esse público (gestantes). Ao se fazer a investigação 
proposta, poderão ser detectados os tipos de TEs mais produzidos, bem como a fase do desenvolvimento a que estas chegam e o assunto do contexto da gestação sobre o qual são produzidas. Sendo assim, para o desenvolvimento deste estudo, o objetivo foi: identificar as Tecnologias Educacionais propostas para gestantes publicadas nos últimos cinco anos.

\section{MÉTODOS}

Optou-se pelo método de Revisão Integrativa para objetivar o sugerido estudo. Souza MT, et al. (2010), classificaram a revisão integrativa de literatura como uma contribuição para determinar o conhecimento atual sobre uma temática específica, de modo a identificar, analisar e sintetizar resultados.

Para o desenvolvimento deste estudo, foi necessário seguir seis fases do processo de elaboração da revisão integrativa. $1^{\underline{a}}$ Fase: elaboração da pergunta norteadora; $2^{\underline{a}}$ Fase: busca ou amostragem na literatura; $3^{\underline{a}}$ Fase: coleta de dados; $4^{\underline{a}}$ Fase: análise crítica dos estudos incluídos; $5^{\underline{a}}$ Fase: discussão dos resultados; 6를 Fase: apresentação da revisão integrativa (SOUZA MT, et al., 2010).

A princípio, delineou-se o tema a se pesquisar: as Tecnologias Educacionais voltadas para gestantes. Embasou-se na estratégia PICoT, onde P - representou a População (Gestantes), I - Intervenção (Tecnologias Educacionais), Co - Contexto (Gestação ou Pré-natal) e T - Cronologia (cinco anos), elaborou-se o seguinte questionamento: Quais as Tecnologias Educacionais propostas para gestantes durante o pré-natal publicadas nos últimos cinco anos?

Os autores realizaram, no período de maio a junho de 2021, a busca na Biblioteca Virtual em Saúde (BVS). As bases de dados selecionadas foram: Medical Literature Analysis and Retrieval System Online (Medline), Literatura Latino-Americana e do Caribe em Ciências da Saúde (Lilacs) e Base de Dados em Enfermagem (Bdenf). Os Descritores em Ciências da Saúde (DeCS) empregados nas buscas foram: Cuidado Pré-Natal (descritor controlado), Gestantes (descritor controlado), Tecnologia Educacional e Educação em Saúde. No Quadro 1, detalhou-se os DeCS, assim como as descrições e seus sinônimos. Também foram utilizadas as palavras-chave: manual, cartilha, aplicativo, guia, caderno, software, jogo, material educativo, álbum seriado, manual educativo, tecnologia educativa. Foram utilizados os operadores booleanos AND e OR e o truncador \$.

Quadro 1 - Descritores em saúde, significados e sinônimos.

\begin{tabular}{|c|l|c|}
\hline $\begin{array}{c}\text { Descritores em } \\
\text { Ciências da Saúde } \\
\text { (DeCS) }\end{array}$ & \multicolumn{1}{|c|}{ Significado } & Sinônimos \\
\hline Cuidado Pré-Natal & $\begin{array}{l}\text { Pré-Natal inclui a prevenção, a promoção da saúde e o } \\
\text { tratamento dos problemas que possam ocorrer durante o } \\
\text { período gestacional e após o parto. A adesão das } \\
\text { mulheres ao pré-natal está relacionada com a qualidade } \\
\text { da assistência prestada pelo serviço e pelos profissionais } \\
\text { de saúde, fator essencial para redução dos elevados } \\
\text { índices de mortalidade materna e perinatal (Assistência } \\
\text { Pré-Natal - Ministério da Saúde, 2020). }\end{array}$ & $\begin{array}{c}\text { Assistência Antenatal; } \\
\text { Assistência Pré-Natal; Pré- } \\
\text { Natal. }\end{array}$ \\
\hline Gestantes & $\begin{array}{l}\text { Mulheres que estão grávidas, como entidades culturais, } \\
\text { psicológicas ou sociológicas. }\end{array}$ & $\begin{array}{c}\text { Grávidas; Mulher Grávida; } \\
\text { Mulheres Grávidas; }\end{array}$ \\
\hline Tecnologia Educacional & $\begin{array}{l}\text { Identificação sistemática, desenvolvimento, organização } \\
\text { ou utilização de recursos educacionais e o manuseio } \\
\text { destes processos. Também é ocasionalmente usada em } \\
\text { um senso mais limitado para descrever o uso das } \\
\text { técnicas orientadas por equipamentos ou auxílio }\end{array}$ & $\begin{array}{c}\text { Tecnologia Instrucional. } \\
\text { audiovisual no cenário educacional. }\end{array}$ \\
\hline Educação em Saúde & $\begin{array}{l}\text { A educação em saúde objetiva desenvolver nas pessoas } \\
\text { um sentido de responsabilidade, como indivíduo, membro } \\
\text { de uma família e de uma comunidade, para com a saúde, } \\
\text { tanto individual como coletivamente. }\end{array}$ & $\begin{array}{c}\text { Educar para a Saúde; } \\
\text { Educação Sanitária; } \\
\text { Educação para a Saúde; } \\
\text { Cománo para a Saúde }\end{array}$ \\
\hline
\end{tabular}

Fonte: Brito AV, et al., 2021. 
Os critérios de inclusão determinados foram: artigos completos, disponíveis on-line, em português e/ou inglês e que abordaram a temática escolhida. O período para corte foi 2016 a 2021 . Os critérios de exclusão foram: publicações nas modalidades cartas, resenhas e editoriais; teses, dissertações, monografia; duplicados; sem convergência com a questão de pesquisa; cujo método não estava bem descrito; com texto completo indisponível e que não foram produzidos para a realidade brasileira.

Por meio desses parâmetros de busca, obtiveam-se 8.084 resultados. Após aplicação de filtro a partir dos critérios de inclusão descritos acima, encontraram-se 1.729 estudos. Após leitura dos títulos, foram excluídos 1.653 por não se relacionarem com o tema desta revisão, seis por não serem do Brasil, um por ser revisão de literatura e 20 por serem duplicados. Ao fim, restaram 49 artigos para leitura dos resumos e posterior seleção de estudos para incluir nos resultados finais desta revisão. Na Figura 1, detalhou-se o fluxo das duas buscas realizadas nessa etapa.

Após a seleção dos artigos para leitura na íntegra, as informações foram extraídas conforme protocolo criado pelos autores.

Figura 1 - Buscas para a revisão.
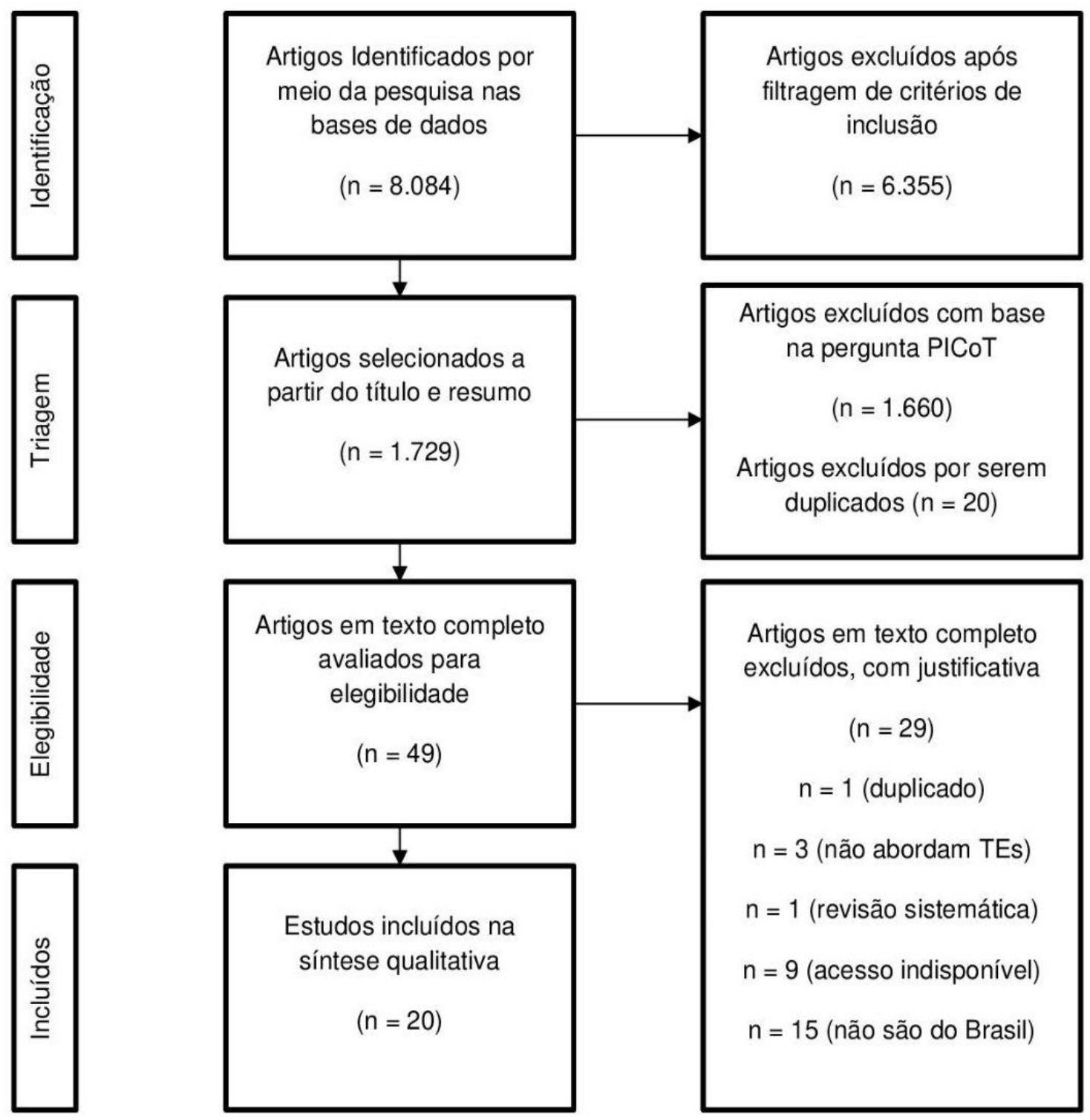

Fonte: Brito AV, et al., 2021. 
Após leitura na íntegra, foram excluídos 29 artigos, destes, um era duplicado, três não tratavam de TE, um era revisão, nove estavam indisponíveis para acesso, 15 artigos não eram do Brasil.

\section{RESULTADOS E DISCUSSÃO}

A amostra final deste estudo foi composta de 20 artigos que foram utilizados para a elaboração dos resultados e discussões. Entre os 20 artigos, dez (50\%) foram publicados em inglês e dez (50\%) em português. Quanto ao público-alvo das TEs, 11 (55\%) eram TEs para gestantes, um (5\%) para gestantes e puérperas e oito (40\%) voltados para profissionais da saúde que utilizariam a tecnologia com as gestantes.

O Quadro 2 apresentou a distribuição dos itens de acordo com o nome dos autores e ano de publicação; título; base de dados e o periódico no qual o estudo foi publicado. Entre as 20 publicações, nove foram do ano de 2019, o que representa $45 \%$ do total. Notou-se a expressiva utilização da base de dados Lilacs (mais de $80 \%$ ) para indexação das revistas nas quais foram publicados os artigos.

No que tange aos periódicos, observou-se que dos 13 periódicos em que os artigos foram publicados, destacaram-se a Rev. Enfermagem UFPE (15\%), Acta Paulista de Enfermagem (15\%) e Revista Brasileira de Enfermagem (15\%), com quase metade das publicações. Destacou-se que $14(70 \%)$ artigos foram publicados em revistas específicas da enfermagem e $12(60 \%)$ artigos foram publicados em revistas classificadas como B1 ou mais, segundo classificação Qualis Capes 2013-2016 (área de avaliação: enfermagem), ou seja, em revistas conceituadas na enfermagem.

No que diz respeito à produção de conhecimento cientifico por parte da enfermagem, Cassiano AN, et al. (2020), explicaram que houve um crescimento exponencial nas últimas décadas com a criação de programas de pós-graduação sritcto sensu e com os grupos de pesquisa em enfermagem que focalizam a temática da tecnologia, permitindo o aumento do número de profissionais mestres e doutores com conhecimento nessa área. Observou-se um crescimento significativo dos anos de 2011 a 2018 de dissertações (144) e teses (42), que abordaram a temática da tecnologia, entre as quais sobressaíram as temáticas relacionadas à enfermagem médico-cirúrgica $(38,5 \%)$, pediátrica $(20,5 \%)$ e obstétrica $(12,8 \%)$.

O Quadro 3 apresentou a distribuição dos itens de acordo com o tipo de estudo desenvolvido, instituição de vinculação dos autores e local de desenvolvimento. Do total dos artigos, destacou-se o uso da pesquisa metodológica com sete (35\%) artigos. Seis (30\%) artigos tiveram entre os autores pessoas com vinculação à Universidade Federal do Ceará. Notou-se que a maioria dos estudos, $13(65 \%)$, foram desenvolvidos somente em Unidades de Atenção Primária à Saúde e apenas quatro $(20 \%)$, desenvolvidos em ambiente hospitalar/Maternidade. Do total de estudos, 13 (65\%) foram realizados em estados da Região Nordeste. 
Quadro 2 - Descrição dos estudos incluídos na revisão integrativa, segundo autor(es), ano, título, base de dados e periódicos de publicação.

\begin{tabular}{|c|c|c|c|c|}
\hline № & Autor/ano & Título & Base de dados & Periódicos \\
\hline 1 & $\begin{array}{l}\text { Santos AL e Souza } \\
\text { MH (2017). }\end{array}$ & Elaboração de novas tecnologias em enfermagem: utilização de uma cartilha para prevenção. & Bdenf & $\begin{array}{l}\text { Revista de Enfermagem UFPE on- } \\
\text { line }\end{array}$ \\
\hline 2 & $\begin{array}{l}\text { Carneiro RF, et al. } \\
(2017) \text {. }\end{array}$ & $\begin{array}{l}\text { Uma nova ferramenta educacional para a prevenção de hipertensão na gravidez - avaliação através } \\
\text { da visita de equipe de saúde da família. }\end{array}$ & Bdenf & $\begin{array}{l}\text { Revista de Enfermagem UFPE on- } \\
\text { line }\end{array}$ \\
\hline 3 & $\begin{array}{l}\text { Ribeiro SC, et al. } \\
\text { (2018). }\end{array}$ & Atividade educativa para a promoção do cuidado com o recém-nascido. & Lilacs & Revista Saúde e Pesquisa \\
\hline 4 & $\begin{array}{l}\text { Oliveira SC, et al. } \\
\text { (2018). }\end{array}$ & Efeito de uma intervenção educativa na gravidez: ensaio clínico randomizado em cluster. & Bdenf e Lilacs & Acta Paulista de Enfermagem \\
\hline 5 & $\begin{array}{l}\text { Javorski M, et al. } \\
(2018) \text {. }\end{array}$ & $\begin{array}{l}\text { Efeitos de uma tecnologia educativa na autoeficácia para amamentar e na prática do aleitamento } \\
\text { materno exclusivo. }\end{array}$ & Bdenf e Lilacs & $\begin{array}{c}\text { Revista da Escola de Enfermagem } \\
\text { da USP }\end{array}$ \\
\hline 6 & $\begin{array}{l}\text { Nunes RD, et al. } \\
(2019) \text {. }\end{array}$ & $\begin{array}{l}\text { Avaliação da efetividade de uma oficina educativa para gestantes com o uso de inquéritos pré e pós- } \\
\text { intervenção. }\end{array}$ & Lilacs & Cadernos de Saúde Pública \\
\hline 7 & $\begin{array}{l}\text { Gomes ML, et al. } \\
\text { (2019). }\end{array}$ & Avaliação de aplicativos móveis para promoção da saúde de gestantes com pré-eclâmpsia. & Bdenf e Lilacs & Acta Paulista de Enfermagem \\
\hline 8 & $\begin{array}{l}\text { Nágila LM, et al. } \\
\text { (2019). }\end{array}$ & $\begin{array}{l}\text { Construção de um mapa de conversação para gestantes e puérperas sobre os cuidados com o } \\
\text { recém-nascido. }\end{array}$ & Lilacs & Revista Cubana de Enfermería \\
\hline 9 & $\begin{array}{l}\text { Silva AB, et al. } \\
(2019) \text {. }\end{array}$ & $\begin{array}{l}\text { Cross-cultural adaptation of the Zero Mothers Die (ZMD App) in Brazil: contributing to digital health } \\
\text { with the approach on care centred for e-pregnant woman. }\end{array}$ & Lilacs & $\begin{array}{l}\text { Revista Brasileira de Saúde } \\
\text { Materno Infantil }\end{array}$ \\
\hline 10 & $\begin{array}{l}\text { Sales RO, et al. } \\
\text { (2019). }\end{array}$ & Desenvolvimento e avaliação de um aplicativo para o controle da sífilis em gestantes. & Bdenf e Lilacs & Revista Brasileira de Enfermagem \\
\hline 11 & $\begin{array}{l}\text { Andrade IS, et al. } \\
\text { (2019). }\end{array}$ & Efeitos de tecnologia no conhecimento, atitude e prática de gestantes para o parto. & Bdenf e Lilacs & Revista Rene \\
\hline 12 & $\begin{array}{l}\text { Silva RM, et al. } \\
\text { (2019). }\end{array}$ & Mobile health technology for gestational care: evaluation of the GestAção's app. & $\begin{array}{l}\text { Bdenf, Lilacs e } \\
\text { Medline }\end{array}$ & Revista Brasileira de Enfermagem \\
\hline 13 & $\begin{array}{l}\text { Antonello VS, et al. } \\
(2019) .\end{array}$ & $\begin{array}{l}\text { The Use of Mobile Educational Tools to Improve Antimicrobial Prescription for the Treatment of Acute } \\
\text { Pyelonephritis in Pregnancy: A Retrospective Cross-sectional Study. }\end{array}$ & Lilacs & $\begin{array}{l}\text { Revista Brasileira de Ginecologia \& } \\
\text { Obstetrícia }\end{array}$ \\
\hline 14 & Rei KL, et al. (2019). & Validação de uma tecnologia educacional: manual obstétrico para a atenção primária. & Bdenf e Lilacs & Revista Nursing \\
\hline 15 & $\begin{array}{l}\text { Hundertmark K, et al. } \\
\text { (2020). }\end{array}$ & Apoia: Desenvolvimento de um protótipo para aplicativo móvel de apoio social à gestação. & Bdenf & $\begin{array}{l}\text { Revista de Enfermagem UFPE on- } \\
\text { line }\end{array}$ \\
\hline 16 & $\begin{array}{l}\text { Santiago RF, et al. } \\
\text { (2020). }\end{array}$ & $\begin{array}{l}\text { Avaliação de objeto virtual de aprendizagem sobre pré-natal para adolescentes grávidas na atenção } \\
\text { básica. }\end{array}$ & Bdenf e Lilacs & Acta Paulista de Enfermagem \\
\hline 17 & $\begin{array}{l}\text { Duque DA e } \\
\text { Fernandes BM } \\
(2020) .\end{array}$ & Construction and validation of a clinical form for usual-risk prenatal follow-up. & Bdenf e Lilacs & Revista de Enfermagem da UFMS \\
\hline 18 & $\begin{array}{l}\text { Facundo SH, et al. } \\
(2020) .\end{array}$ & Communication technologies used by nurses in prenatal care. & Lilacs & $\begin{array}{l}\text { Revista Brasileira em Promoção da } \\
\text { Saúde }\end{array}$ \\
\hline 19 & $\begin{array}{l}\text { Souza FM, et al. } \\
(2021) \text {. }\end{array}$ & $\begin{array}{l}\text { Effectiveness of mobile applications in pregnant women's adherence to prenatal consultations: } \\
\text { randomized clinical trial. }\end{array}$ & Bdenf e Lilacs & Revista Brasileira de Enfermagem \\
\hline 20 & $\begin{array}{l}\text { Queiroz FF, et al. } \\
\text { (2021). }\end{array}$ & Evaluation of the 'Gestação' application from the perspective of semiotics: pregnant women's views. & $\begin{array}{l}\text { LILACS e } \\
\text { MEDLINE }\end{array}$ & Revista Ciência \& Saúde Coletiva \\
\hline
\end{tabular}

Fonte: Brito AV, et al., 2021. 


\begin{tabular}{|c|c|c|c|}
\hline № & Tipo de Estudo & Instituição dos Autores & Local \\
\hline 1 & Qualitativo & Centro Universitário Franciscano/RS & Maternidade em Santa Maria/RS \\
\hline 2 & Metodológico e quantitativo & $\begin{array}{l}\text { Universidade Estadual do Ceará/ CE; Universidade Federal da Bahia/BA; Universidade de } \\
\text { Fortaleza/CE }\end{array}$ & $\begin{array}{l}\text { Unidades } \\
\text { Primária à Saúde, em Fortaleza/CE }\end{array}$ \\
\hline 3 & $\begin{array}{l}\text { Estudo exploratório, descritivo e } \\
\text { quantitativo }\end{array}$ & $\begin{array}{l}\text { Centro Universitário Estácio do Ceará/CE Prefeitura Municipal de Mombaça/CE; } \\
\text { Universidade Estadual de Campinas/SP; Universidade Federal do Piauí/PI; Universidade } \\
\text { Federal da Paraíba/PB }\end{array}$ & Maternidade em Mombaça-CE \\
\hline 4 & Ensaio Clínico randomizado & Universidade Federal de Pernambuco/PE Universidade Federal do Ceará/CE & Unidades de Saúde de Recife \\
\hline 5 & $\begin{array}{l}\text { Estudo de intervenção, controlado e } \\
\text { randomizado }\end{array}$ & $\begin{array}{l}\text { Universidade Estadual do Ceará/CE; Universidade } \\
\text { Universidade Federal do Ceará/CE }\end{array}$ & Unidades Básicas de Saúde de Recife \\
\hline 6 & $\begin{array}{l}\text { Estudo Transversal, com delineamento } \\
\text { de pesquisa pré e pós-intervenção }\end{array}$ & Universidade do Sul de Santa Catarina, Palhoça/SC & $\begin{array}{l}\text { Serviço da Universidade do Sul de Santa } \\
\text { Catarina, na cidade de Palhoça, SC }\end{array}$ \\
\hline 7 & Estudo Descritivo & $\begin{array}{l}\text { Universidade Federal do Ceará/CE; Universidade Federal do Piauí/PI; Secretaria Municipal } \\
\text { de Acarape /CE; Prefeitura Municipal de Fortaleza/CE }\end{array}$ & Plataformas de venda de aplicativos digitais \\
\hline 8 & Estudo Metodológico & Universidade Federal de Pernambuco/PE Universidade Federal do Ceará/CE & $\begin{array}{l}\text { Unidade de Extensão da Universidade Federal do } \\
\text { Ceará }\end{array}$ \\
\hline 9 & $\begin{array}{l}\text { Estudo descritivo de natureza } \\
\text { exploratória }\end{array}$ & $\begin{array}{l}\text { Fundação Oswaldo Cruz/RJ; Instituto Fernandes Figueira/RJ; Instituto Nacional de Saúde da } \\
\text { Mulher, da Criança e do Adolescente Fernandes Figueira/RJ; Universidade Federal do Rio } \\
\text { de Janeiro/RJ }\end{array}$ & $\begin{array}{l}\text { Instituto Nacional de Saúde da Mulher, da Criança } \\
\text { e do Adolescente Fernandes Figueira, localizado } \\
\text { na cidade do Rio de Janeiro }\end{array}$ \\
\hline 10 & Pesquisa Metodológica & Universidade de Fortaleza/CE & $\begin{array}{l}\text { Unidades Básicas de Saúde e Hospitais de } \\
\text { referência no atendimento a gestantes no } \\
\text { Município de Fortaleza-Ceará }\end{array}$ \\
\hline 11 & Estudo quase Experimental & Universidade Federal do Ceará/CE & $\begin{array}{l}\text { Unidades básicas de saúde da família no } \\
\text { município de Eusébio, Ceará, Brasil. }\end{array}$ \\
\hline 12 & $\begin{array}{l}\text { Estudo de natureza avaliativa, aplicada, } \\
\text { metodológica, com abordagem quanti- } \\
\text { qualitativa. }\end{array}$ & Universidade de Fortaleza/CE & $\begin{array}{l}\text { Unidades de Atenção Primária à Saúde (Uaps), } \\
\text { município de Fortaleza, Ceará, Brasil }\end{array}$ \\
\hline 13 & Estudo transversal retrospectivo & $\begin{array}{l}\text { Hospital Fêmina, Porto Alegre/RS; Universidade do Vale do Rio dos Sinos, São } \\
\text { Leopoldo/RS; Universidade Federal do Rio Grande do Sul, Porto Alegre/RS; Pontifícia } \\
\text { Universidade Católica do Rio Grande do Sul, Porto Alegre/ RS }\end{array}$ & $\begin{array}{l}\text { Hospital Fêmina, em Porto Alegre, no estado do } \\
\text { Rio Grande do Sul }\end{array}$ \\
\hline 14 & $\begin{array}{l}\text { Pesquisa aplicada, de natureza } \\
\text { experimental }\end{array}$ & Universidade Estadual do Pará/PA & Unidades Básicas de Saúde (UBSs)- Marabá-PA \\
\hline 15 & Estudo misto transversal & Universidade Federal de Santa Maria/RS; Universidade Franciscana/RS & $\begin{array}{l}\text { Serviços de atenção básica à saúde do município } \\
\text { de Santa Maria, Rio Grande do Sul. }\end{array}$ \\
\hline 16 & $\begin{array}{l}\text { Estudo Metodológico, tecnológico de } \\
\text { abordagem quantitativo }\end{array}$ & $\begin{array}{l}\text { Universidade Estadual do Piauí/PI; Universidade Regional do Cariri/CE; Universidade de } \\
\text { São Paulo/SP }\end{array}$ & Unidades Básicas de Saúde (UBSs) de Teresina \\
\hline 17 & $\begin{array}{c}\text { Pesquisa Metodológica com Técnica } \\
\text { Delphin }\end{array}$ & Universidade Federal de Juiz de Fora/MG & De caráter virtual - âmbito Nacional \\
\hline 18 & Descritivo transversal & $\begin{array}{l}\text { Universidade de Fortaleza/CE; Universidade Estadual do Ceará/CE; Centro Universitário } \\
\text { Estácio do Ceará/CE }\end{array}$ & $\begin{array}{l}\text { Centros de Atenção } \\
\text { Saúde de Fortaleza, Ceará }\end{array}$ \\
\hline 19 & Ensaio clínico randomizado & Universidade Federal do Rio Grande do Norte/RN & $\begin{array}{l}\text { Unidades de Saúde da Família (USFs) no } \\
\text { Nordeste }\end{array}$ \\
\hline 20 & $\begin{array}{l}\text { Natureza aplicada, metodológica, com } \\
\text { abordagem qualitativa }\end{array}$ & Universidade de Fortaleza/CE; Universidade Federal do Ceará/CE & $\begin{array}{l}\text { Unidades de Atenção Primária à Saúde (Uaps) na } \\
\text { cidade de Fortaleza, Ceará }\end{array}$ \\
\hline
\end{tabular}
Fonte: Brito AV, et al., 2021. 
Melo WS, et al. (2017), explicaram que os estudos metodológicos se referiram ao desenvolvimento, validação e avaliação de ferramentas e métodos de pesquisa. O aumento da preferência pela pesquisa metodológica entre enfermeiros pesquisadores se deve a análise de resultados consistentes e exatos, testes rigorosos de intervenção e procedimentos aprimorados de obtenção de dados.

Nos resultados, no que diz respeito à vinculação dos autores, notou-se que há uma concentração de autores da região Nordeste. O estudo de Cassiano AN, et al. (2020), revelou que o Nordeste é a região do país que mais desenvolve pesquisas metodológicas, principalmente as que objetivam validar tecnologias. A Universidade Federal do Ceará tem o Programa de Pós-Graduação em Tecnologia Educacional (mestrado) e a Universidade de Fortaleza, o Mestrado Profissional em Tecnologia e Inovação em Enfermagem.

Além disso, Sidone OJ, et al. (2016), relataram que, por muitos anos, a região Sudeste esteve em evidência no que tange às produções científicas no geral, devido às suas instituições e incentivos à pesquisa que ali fomentam e acabam facilitando o processo de produções e publicações, porém, no decorrer dos anos, notou-se uma desconcentração com uma participação crescente e expressiva de autores da região Nordeste e Sul.

Silva DM, et al. (2017), disseram que, com a globalização, o processo educativo no âmbito da saúde requer inovações. A educação em saúde é "definida como combinações de experiências de aprendizagem; e delineada com o objetivo de facilitar ações voluntárias que conduzem à saúde" (p. 1.045). As tecnologias educacionais são ferramentas úteis e importantes que podem ser utilizadas no processo de ensino no contexto da assistência de enfermagem, sendo um meio facilitador para desenvolver a educação em saúde e promover conhecimento e saúde à população.

Entretanto, Figueira AB, et al. (2013), no estudo que abordou a visão do enfermeiro acerca da prática da educação em saúde no ambiente hospitalar, observou-se que o pouco incentivo nas formações dos profissionais enfermeiros sobre a temática acaba cerceando a educação em saúde, pois os enfermeiros praticam as atividades voltadas para educação em saúde dentro das redes hospitalares, porém, não as compreendem como tais, ou consideram que ação educativa é de responsabilidade somente da atenção primária. Outra dificuldade apontada pelos profissionais da área hospitalar foi a sobrecarga de trabalho. Sendo assim, tal achado é uma possível resposta para o porquê de, neste estudo, terem sido encontradas mais tecnologias voltadas para o âmbito da atenção primária.

O Quadro 4 apresentou a distribuição dos itens de acordo com o tipo de tecnologia que foi desenvolvida, o assunto abordado e em qual fase de desenvolvimento está (construção, validação, aplicação ou levantamento das tecnologias). Evidenciou-se que o tipo de tecnologia mais utilizado foi Aplicativo Móvel em oito (40\%) estudos. Já referente aos assuntos mais abordados, verificou-se que não houve predominância de um assunto, os temas foram diversificados, como direito da mulher, hipertensão, cuidados ao recémnascido, alimentação, amamentação, entre outros. Quanto ao tipo de desenvolvimento, aplicação (sete estudos) e validação (cinco estudos só validação e três construção e validação) foram os que mais se destacaram. 
Quadro 4 - Descrição dos estudos incluídos na revisão integrativa, segundo tipo de tecnologia, assunto da tecnologia e fase do desenvolvimento da pesquisa.

\begin{tabular}{|c|c|c|c|}
\hline No. & Tipo de tecnologia & Assunto da tecnologia & Fase do desenvolvimento \\
\hline 1 & Cartilha & Direitos da mulher no pré-natal, parto e pós-parto (violência obstétrica). & Construção \\
\hline 2 & Guia & Hipertensão na gravidez & Validação \\
\hline 3 & $\begin{array}{l}\text { Atividade educativa com álbum } \\
\text { seriado e folders. }\end{array}$ & Cuidados e segurança do Recém-Nascido, Aleitamento Materno. & Aplicação \\
\hline 4 & $\begin{array}{l}\text { Intervenção Educativa com } \\
\text { Cartilha }\end{array}$ & Alimentação saudável com uso de alimentos regionais. & Aplicação \\
\hline 5 & Álbum seriado & Aleitamento materno exclusivo (AME) & Aplicação \\
\hline 6 & Oficina & $\begin{array}{l}\text { Conhecimentos sobre a gravidez, o parto, a amamentação e os cuidados com o } \\
\text { recém-nascido. }\end{array}$ & Aplicação \\
\hline 7 & Aplicativos móveis & Pré-eclâmpsia (PE) & Levantamento \\
\hline 8 & Mapa de Conversação & $\begin{array}{l}\text { Tecnologia educativa sobre os cuidados com o recém-nascido para gestantes e } \\
\text { puérperas }\end{array}$ & Construção \\
\hline 9 & Aplicativo Móvel & Combate à mortalidade materna & Validação \\
\hline 10 & Aplicativo Móvel & Controle da sífilis em gestantes & Construção e Validação \\
\hline 11 & Vídeo educativo & Conhecimento, a atitude e a prática de gestantes para o parto ativo & Aplicação \\
\hline 12 & Aplicativo Móvel & Cuidados com a saúde gestacional & Validação \\
\hline 13 & Aplicativo Móvel & Pielonefrite aguda & Aplicação \\
\hline 14 & Manual Obstétrico & Informações sobre a consulta no pré-natal & Construção e Validação \\
\hline 15 & Aplicativo móvel & Apoio social para as gestantes & Construção \\
\hline 16 & Objeto Virtual de Aprendizagem & Pré-natal para adolescentes grávidas no contexto da atenção básica & Validação \\
\hline 17 & Ficha Clínica & Acompanhamento do pré-natal de risco habitual & Construção e Validação \\
\hline 18 & $\begin{array}{c}\text { Tecnologias de comunicação: } \\
\text { prontuário eletrônico e ficha de } \\
\text { pré-natal }\end{array}$ & Otimização na assistência do Profissional de Saúde e paciente & Levantamento de tecnologias utilizadas \\
\hline 19 & Aplicativo Móvel & Adesão de gestantes às consultas de pré-natal & Aplicação \\
\hline 20 & Aplicativo Móvel & As fases do período gestacional & Validação \\
\hline
\end{tabular}

Fonte: Brito AV, et al., 2021. 
O avanço das tecnologias voltadas para a saúde e o aumento da aquisição de telefones celulares/smartphones têm contribuído para melhoria das condições de saúde de diversos grupos. Muitos usam o smartphone como o principal meio para acessar informações, além de ferramenta para 0 gerenciamento da saúde. Esses dispositivos móveis com suas tecnologias (telefonia, textos, vídeos, internet e aplicativos para smartphones) transformaram o dia a dia de muitas pessoas, possibilitando experiências diferenciadas de aprendizagem e entretenimento, fomentando o cuidado em saúde e a aquisição de conhecimento pelos usuários, profissionais e pesquisadores. Assim, há diversas possibilidades com uso dessas tecnologias em favor da promoção da saúde nos mais diversos contextos, incluindo a gestação e assim corroborou com os achados do presente estudo (SILVA RM, et al., 2019).

Quanto aos assuntos abordados, embora tenham sido diversificados, ainda sim, segundo Mota FR, et al. (2015), há necessidade de que as gestantes tenham suas dúvidas sanadas, com informações mais diretas, com uso de materiais de linguagem mais acessível ao nível socioeconômico dessas gestantes (os autores dão como exemplo o uso de folder) e que estejam disponíveis na própria unidade de atendimento. Os autores detectaram deficiência nas informações repassadas às gestantes e apontaram o quanto a falta de conhecimento destas reflete na saúde delas mesmas e do feto.

Como exemplo de assuntos que podem ser abordados, estão as infecções sexualmente transmissíveis, que podem trazer potenciais complicações para a gestação e o feto (MOURA RJ, et al., 2018). No que concerniu à temática da diabetes mellitus gestacional (DMG), Schoenaker DA, et al. (2016), ressaltaram que houve uma elevação de sua prevalência, junto com o aumento da prevalência de obesidade. Mostrando assim que diabetes gestacional, alimentação, hábitos de vida saudáveis também precisam ser abordados com as gestantes.

Além disso, é importante a abordagem da saúde mental das gestantes, pois, segundo Guimarães FJ, et al. (2019), a depressão pós-parto e o bem-estar mental durante todo o processo materno requer acompanhamento e estudos, que visem reconhecer o adoecimento mental durante a gravidez, como também orientar as gestantes e profissionais sobre essa problemática. Assim, apontaram-se inúmeras outras possibilidades de desenvolvimento de TE para gestantes.

O desenvolvimento de uma TE é constituído em três fases: construção, validação e aplicação. Teixeira $E$ e Nascimento HM (2020), discutiram três vias para construção de uma TE: na primeira via, a construção é baseada apenas na literatura, com revisão da literatura, em que se destacou a revisão integrativa como mais profícua; na segunda via, a construção é baseada no contexto, em que é feita a revisão de literatura e essa é complementada por uma escuta sensível do público-alvo e o pesquisador constrói a TE; e, na terceira via, há a revisão de literatura e escuta sensível da segunda via, mas quem constrói a TE é o pesquisador e o público-alvo.

Moreira AP, et al. (2014), explicaram que o processo de validação tem como objetivo investigar o estágio em que um instrumento se expressa apropriado para verificar o que ele provavelmente deveria ponderar, ou seja, é uma estratégia que visa criar uma nova intervenção/instrumento ou aprimorar um já existente. Ela deve ser avaliada por um grupo de juízes ou peritos com experiência na área do assunto, o qual deverá avaliar os itens e determinar se eles são inclusivos e essenciais para o que se deseja medir. Podem ser validados conteúdo, critério e/ou constructo. Já a aplicação da tecnologia, de acordo com Silva AP, et al. (2017), verificou-se a efetividade da estratégia.

Nota-se que, com o crescimento da temática das TEs, as fases também foram avançando e muitas estão na última etapa do desenvolvimento (as aplicações) e que assim a efetividade dessas tecnologias poderá ser apresentada para o público-alvo (as gestantes).

Nos estudos encontrados nesta revisão, diferentemente das fases mencionadas anteriormente, dois fizeram levantamentos e um pesquisou os aplicativos móveis para promoção da saúde de gestantes como pré-eclâmpsia (GOMES ML, et al., 2019). Um estudo objetivou identificar as tecnologias de comunicação que os profissionais enfermeiros utilizavam no pré-natal prestado na atenção primária, e seus autores, Facundo SB, et al. (2020), enfatizaram que poucas tecnologias são utilizadas para aproximação dos profissionais enfermeiros e/ou equipe multiprofissional e as gestantes. 
Como limitação do estudo apontou-se o fato de o levantamento ter sido realizado apenas com referência aos últimos cinco anos e somente com artigos, descartando teses e dissertações. Entretanto, essa escolha metodológica pautou-se na necessidade de discutir as evidências mais atualizadas e de maior circulação acadêmico-científica e no fato de haver uma recente publicação de estudo bibliométrico em teses e dissertações de enfermagem, com foco na validação de tecnologias (CASSIANO AN, et al., 2020).

\section{CONSIDERAÇÕES FINAIS}

Evidenciou-se um aumento de publicações e o protagonismo da enfermagem quanto à construção, validação e aplicação de Tecnologias Educacionais. Entretanto, estas ainda são escassas acerca do contexto de atenção ao pré-natal. Houve uma concentração de estudos realizados por pesquisadores de instituições do Nordeste (principalmente do Ceará) e voltados para a realidade dessa região, apontando possibilidades e a necessidade de redes de colaboração científica, mas também a carência tanto de produção científica como de foco dos estudos para o contexto da saúde das regiões Norte e Centro-Oeste. Por fim, mostrou-se necessário o incentivo para estudos também voltados para o contexto hospitalar, que precisa ser valorizado e compreendido como um local de educação em saúde, mediado pelo uso de TEs.

\section{REFERÊNCIAS}

1. ANDRADE IS, et al. Efeitos de Tecnologia no conhecimento, atitude e pratica de gestantes para o parto. Revista da Rede de Enfermagem do Nordeste, 2019; 20: e41341.

2. ANTONELLO VS, et al. The Use of Mobile Educational Tools to Improve Antimicrobial Prescription for the Treatment of Acute Pyelonephritis in Pregnancy: A Retrospective Cross-sectional Study. Revista Brasileira de Ginecologia e Obstetrícia, 2019; 41(2): 97-101.

3. CARNEIRO RF, et al. Uma nova ferramenta educacional para a prevenção de hipertensão na gravidez - avaliação através da visita de equipe de saúde da família. Revista de Enfermagem UFPE on line, 2017; 11(3): 1439-1450.

4. CASSIANO AN, et al. Validação de tecnologias educacionais: estudo bibliométrico em teses e dissertações de enfermagem. Revista de Enfermagem do Centro-Oeste Mineiro, 2020; 10: e3900.

5. DUQUE DA, FERNANDES BM. Construction and validation of a clinical form for usual-risk prenatal follow-up. Revista de Enfermagem da UFSM, 2020; 10: 1-16.

6. FACUNDO SH, et al. Communication technologies used by nurses in prenatal care. Revista Brasileira em Promoção da Saúde, 2020; 33: 1-9.

7. FIGUEIRA $A B$, et al. Visão do enfermeiro frente à prática da educação em saúde no ambiente hospitalar. Cogitare Enfermagem, 2013; 18(2): 310-316.

8. GOMES ML, et al. Avaliação de aplicativos móveis para promoção da saúde de gestantes com pré-eclâmpsia. Acta Paulista de Enfermagem, 2019; 32(3): 275-81.

9. GUIMARÃES FJ, et al. Enfermedad mental en mujeres embarazadas. Enfermería Global, 2019; 18(1): 499-534.

10. HUNDERTMARCK K, et al. Apoia: Desenvolvimento de um protótipo para aplicativo móvel de apoio social à gestação. Revista de Enfermagem UFPE on line, 2020; 15(1): e244466.

11. INDARA CB, et al. Tecnologia Educativa para gestantes: Construção e Avaliação de Cartilha. Convención Internacional de Salud, 2018; 11(2): 1-8.

12. JARDIM MJ, et al. Contribuições do Enfermeiro no Pré-Natal para a Conquista do Empoderamento da Gestante. Revista de Pesquisa Cuidado é Fundamental Online, 2019; 11(2, n. esp): 432-440.

13. JAVORSKI M, et al. Efeitos de uma tecnologia educativa na autoeficácia para amamentar e na prática do aleitamento materno exclusivo. Revista da Escola de Enfermagem da USP, 2018; 52: e03329.

14. LIMA VK, et al. Educação em saúde para gestantes: a busca pelo empoderamento materno no ciclo gravídicopuerperal. Revista de Pesquisa Cuidado é Fundamental Online, 2019; 11(4): 968-975.

15. MELO WS, et al. Guide of attributes of the nurse's political competence: a methodological study. Revista Brasileira de Enfermagem, 2017; 70(3): 526-34.

16. MOREIRA AP, et al. Jogo educativo de administração de medicamentos: um estudo de validação. Revista Brasileira de Enfermagem, 2014; 67(4): 528-534.

17. MOTA FR, et al. Necessidade informacionais das gestantes atendidas em unidades básicas de saúde do bairro Benedito Bentes - Maceió/AL. Encontro Nacional de Pesquisa em Ciência da Informação, 2017; 16(11): 1-16.

18. MOURA RJ, et al. Fatores relacionados ao não uso de medidas preventivas das infecções sexualmente transmissíveis durante a gestação. Revista Baiana de Enfermagem, 2018; 32: e26104. 
19. NÁGILA LM, et al. Construção de um mapa de conversação para gestantes e puérperas sobre os cuidados com o recém-nascido. Revista Cubana de Enfermería, 2019; 35(2).

20. NUNES RD, et al. Avaliação da efetividade de uma oficina educativa para gestantes com o uso de inquéritos pré- e pós-intervenção. Cadernos de saúde pública, 2019; 35(10): 1-7.

21. OLIVEIRA SC, et al. Efeito de uma intervenção educativa na gravidez: ensaio clínico randomizado em cluster. Acta Paulista de Enfermagem, 2018; 31(3): 291-298.

22. QUEIROZ FF, et al. Evaluation of the 'Gestação' application from the perspective of semiotics: pregnant women's views. Ciência \& Saúde Coletiva, 2021; 26(2): 485-492.

23. REI KL, et al. Validação de uma tecnologia educacional: manual obstétrico para a atenção primária. Revista Nursing, 2019; 22(258): 3351-3355.

24. RIBEIRO SC, et al. Atividade educativa para a promoção do cuidado com o recém-nascido. Revista Saúde e Pesquisa, 2018; 11(3): 545-553.

25. SABINO LM, et al. Uso de tecnologia leve-dura nas práticas de enfermagem: análise de conceito. Aquichan, 2016; 16(2): 230-239.

26. SALBEGO C, et al. Care-educational technologies: an emerging concept of the praxis of nurses in a hospital context. Revista Brasileira de Enfermagem, 2018; 71(6): 2666-2674.

27. SALES RO, et al. Desenvolvimento e avaliação de um aplicativo para o controle da sífilis em gestantes. Revista Brasileira de Enfermagem, 2019; 72(5): 1326-1332.

28. SANTIAGO RF, et al. Avaliação de objeto virtual de aprendizagem sobre pré-natal para adolescentes grávidas na atenção básica. Acta Paulista de Enfermagem, 2020; 33: eAPE20190063.

29. SANTOS AL, SOUZA MH. Elaboração de novas tecnologias em enfermagem: utilização de uma cartilha para prevenção. Revista de Enfermagem UFPE on line, 2017; 11(10): 3893-3898.

30. SCHOENAKER DA, et al. The role of energy, nutrients, foods, and dietary patterns in the development of gestational diabetes mellitus: a systematic review of observational studies. Diabetes Care, 2016; 39(1): 16-23.

31. SIDONE OJ, et al. A ciência nas regiões brasileiras: evolução da produção e das redes de colaboração científica. Transinformação, 2016; 28(1): 15-32.

32. SILVA AB, et al. Cross-cultural adaptation of the Zero Mothers Die (ZMD App) in Brazil: contributing to digital health with the approach on care centred for e-pregnant woman. Revista Brasileira de Saúde Materno Infantil, 2019; 19(4): 763-775.

33. SILVA AP, et al. Efeitos da aplicação de uma tecnologia educativa na detecção precoce do câncer de mama. Revista da Rede de Enfermagem do Nordeste, 2017; 18(3): 404-11.

34. SILVA DM, et al. Tecnologias educacionais na assistência de enfermagem em educação em saúde: revisão integrativa. Revista enfermagem UFPE online, 2017; 11(Supl. 2): 1044-51.

35. SILVA RM, et al. Mobile health technology for gestational care: Evaluation of the GestAção's app. Revista Brasileira de Enfermagem, 2019; 72(Suppl 3): 266-273.

36. SOUZA FM, et al. Effectiveness of mobile applications in pregnant women's adherence to prenatal consultations: randomized clinical trial. Revista Brasileira de Enfermagem, 2021; 74(Suppl 5): e20190599.

37. SOUZA MT, et al. Revisão Integrativa: o que é e como fazer. Einstein, 2010; 8(1): 102-106.

38. TEIXEIRA E, Nascimento HM. Revisão Integrativa: etapa fundamental para construção baseada na literatura de tecnologias cuidativo-educacionais. In: Desenvolvimento de tecnologias cuidativo-educacional. Porto Alegre: MORIÁ Editora, 2020; 2: 393p. 